\title{
Channel Estimation Techniques for OFDM and UFMC Systems for 5G Communications
}

\author{
Tipparti Anil Kumar, Sk Nilofer, Rajidi Sahithi
}

\begin{abstract}
In this paper, pilot-assisted techniques for channel estimation (CE) are simulated for Universal Filtered Multi-Carrier (UFMC) modulation scheme. UFMC aims at replacing orthogonal frequency division multiplexing (OFDM) and improves performance and robustness in the case of timefrequency misalignment. These techniques efficiently support Internet of Things (IoT) and massive machine type communications (mMTC), which are identified as challenges for $5 G$ wireless communication systems (WCS). Pilot-aided techniques are adopted and applied to OFDM and UFMC. Simulation results are supplemented to compare the performance of UFMC systems with conventional CP-OFDM systems.

Index terms:-Channel estimation; 5G; timing offset; synchronization; OFDM; UFMC;
\end{abstract}

\section{INTRODUCTION}

A completesystemredesignisneededfornext generation $5 \mathrm{GWCS}$ to support rapidgrowth in M2McommunicationsandofIoT [1].In order to minimize the signaling overhead and battery and power consumption for low-end devices like simple sensor elements in 5G, they should be transmitted with synchronization conditions relaxed w.r.t time-frequency misalignments [2].

\section{AsOFDM}

sensitivetothesemisalignmentsduetohighspectralside-

lobelevel [3], andFilter-bankbasedmulticarrier(FBMC)[4]isdisadvantageous forcommunication in short uplink bursts (as required in possible application situations of $5 \mathrm{G}$ systems, for example low latency communication or energy-efficient mMTC, UFMC [58], which may be assumed as commonality of filtered OFDM and FBMC, is proposed forfuture 5GWCS. In this paper, the $\mathrm{CE}$ techniques are analyzed and compared OFDM and UFMC systems for 5G. Channel estimation techniques are discussed related to different channel models and different scenarios with different aspects. Simulation results are shown and performance comparison is made with respect to system parameters.

\section{MULTI-CARRIER MODULATIONTECHNIQUES}

Multicarriersystemsadvantageousover single carrier systems in terms of spectral efficiency [9], datarate,

Revised Manuscript Received on August 19, 2019.

Dr. Tipparti Anil Kumar, Department of Electronics \& Communication Engineering, CMR Institute of Technology, Kandlakoya (v), Medchal Road, Hyderabad - 501401. (tvakumar2000@yahoo.co.in)

Sk Nilofer, Department of Electronics \& Communication Engineering, CMR Institute of Technology, Kandlakoya (v), Medchal Road, Hyderabad -501401. (nilofershaik@gmail.com)

Rajidi Sahithi, Department of Electronics \& Communication Engineering, CMR College of Engineering \& Technology, Kandlakoya (v), Medchal Road, Hyderabad - 501401. (sahithiramu@gmail.com) immunity to frequency-selective and non- selective channels, and adaptive power allocation etc,.

\section{2. a. Universal Filtered Multi-Carrier}

The UFMC system model is shown in Fig. 1

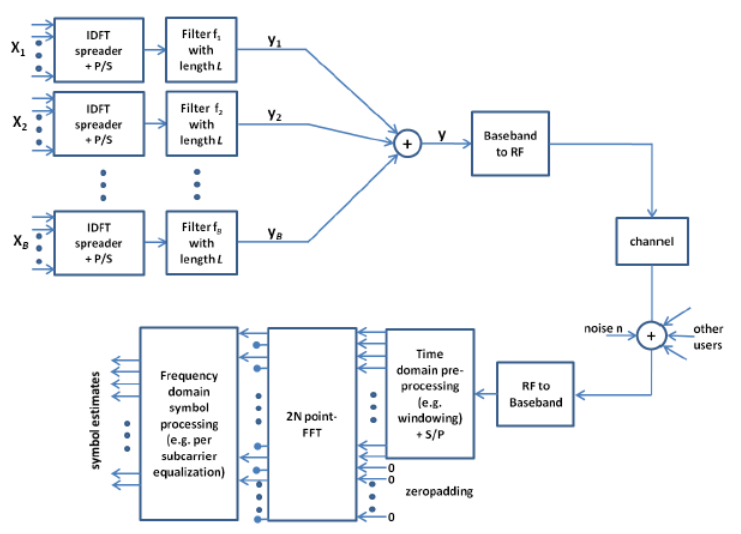

Fig.1: System model of UFMC.

The system bandwidth is sub-divided into number of B sub-bands. The every sub-band is represented as number of physical resource element in LTE system $N_{B}, N$ is the total subcarriers. $N$-point IDFT is performed to transform signal from frequency to time domain. Symbolsaremodulatedandzerosarepaddedin frequency domain to perform IDFT. The signal $x_{i}$ is filtered by FIRfilter $f_{i}$ of $L$. Therefore,sub bandioutputwith symbol length $\mathrm{N}+\mathrm{L}-1$ (linear convolution of $x_{i}$ and $f_{i}$ ) isgivenby

$$
y_{i}(k)=x_{i} * f_{i}=\sum_{l=o}^{L-1} f_{i}(l) x_{i}(k-l), \quad k=0,1, \ldots
$$

\section{Single- user channel estimation}

If channel assisted ISI is negligible, then the channel estimates for given signal $X(k)$ for OFDM and UFMC at sub carrier $k$ maybe expressed as eq. (1) and eq. (2) respectively [6]

$$
\hat{H}(k)=\frac{Y_{o}(k)}{X(k)}=H(k)+\frac{N(k)}{X(k)}
$$




$$
\hat{H}(k)=\frac{Y_{U}(k)}{X_{i}(k) F_{i}(k)}=H(k)+\frac{N(k)}{X_{i}(k) F_{i}(k)}
$$

where $Y(k)$ is the received signal, $N(k)$ is AWGN and $F_{i}(k)$ is frequency response of FIR filter.

Therefore, the CE of UFMC systems differs in the equalization of shape of the filter. Mathematically, final channel estimates for single user systems with sliding window algorithm may be given as [7]

\section{MULTIUSER CHANNEL ESTIMATION}

A general received signal, if all users are devoted with $N_{\text {Alloc }}$ subcarriers, in OFDM is given by

$$
Y_{O}=S_{O}^{/} H+W_{O}
$$

where $H=\left[H_{0}, H_{1}, \ldots . ., H_{N_{\text {user }}-1}\right] \quad$ is channel information vector of size $N_{\text {user }} N_{\text {Alloc }} X 1$ in the respective subcarrier position for all active users. $\mathrm{W}_{\mathrm{O}}$ is noise vector of size $N_{\text {Alloc }} X 1$ and

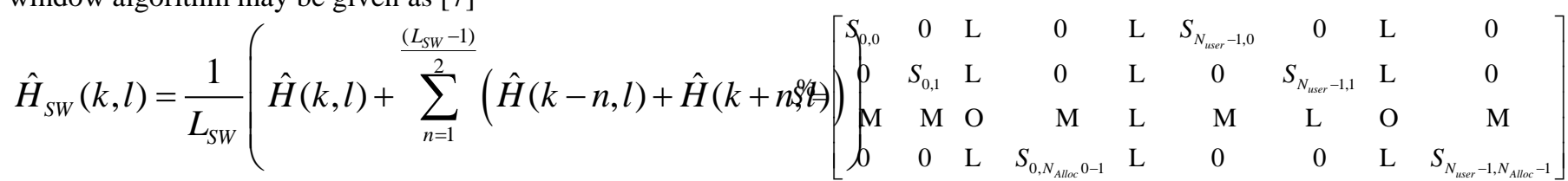

where $\mathrm{L}_{\mathrm{SW}}$ is size of the selection window.

In simulations, flat-fading channel is considered, simulation parameters FFT size 1024, Filter/ CP length: 74/ 73, PRB size: 12, No. of PRBs: 10, Modulation: QPSK and speed of the user is $50 \mathrm{kmh}$. Symbol error rate (SER) vsEb/ No are simulated in Fig. 1 and 2. From simulation results it is observed that SER reduces as the sliding window size increases. According to the obtained SER values, size of sliding window of 7 or 9 may be selected for flat-fading channel model.

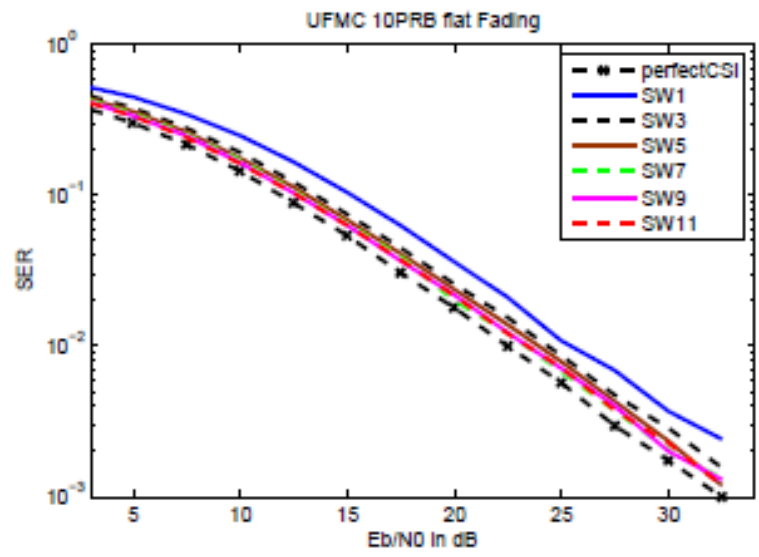

Fig.1.: Single user performance for sliding window UFMC channel estimator.

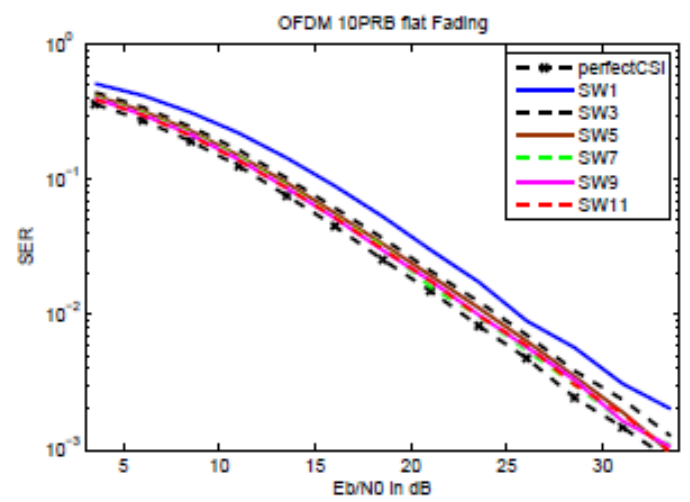

Fig.2.: Single user performance for sliding window OFDM channel estimator
Similarly, the UFMC the received signal can be expressed as

$Y_{U}=\$_{U}^{/ o} H+W_{U}$

Filter shape is also considered in $S_{U}^{o}$ with

$\xi_{U}^{o}=F / S_{O}^{O}$

where e stands for Hadamard-product of matrices.

Mathematically, final channel estimates for multiuser systems for OFDM and UFMC systems taking sliding window algorithm into account may be written as

$$
\hat{H}=S^{+} Y_{O, U}=H+S^{+} W_{O, U}
$$

where $S^{+}\left(=S^{H}\left(S S^{H}\right)^{-1}\right)$ is Moore-Penrose inverse of $S$ for both UFMC and OFDM systems and

for OFDM

$$
S=\left[\begin{array}{ccc}
S_{0, i} & \mathrm{~L} & S_{N_{\text {user }}-1, i} \\
S_{0, i+1} & \mathrm{~L} & S_{N_{\text {user }}-1, i+1} \\
\mathrm{M} & \mathrm{O} & \mathrm{M} \\
S_{0, i+L_{S W}-1} & \mathrm{~L} & S_{N_{\text {user }}-1, i+L_{S W}-1}
\end{array}\right]
$$

and

$S=\left[\begin{array}{ccc}F_{0, i} S_{0, i} & \mathrm{~L} & F_{N_{\text {user }}-1, i} S_{N_{\text {user }}-1, i} \\ F_{0, i+1} S_{0, i+1} & \mathrm{~L} & F_{N_{\text {user }}-1}, i+1 S_{N_{\text {user }}-1, i+1} \\ \mathrm{M} & \mathrm{O} & \mathrm{M} \\ F_{0, i+L_{S W}-1} S_{0, i+L_{S W}-1} & \mathrm{~L} & F_{N_{\text {user }}-1, i+L_{S W}-1} S_{N_{\text {user }}-1, i+L_{S W}-1}\end{array}\right]$

for UFMC 
Combination of DFT-sequence and sliding window, the performance of single and two UEs are examined for frequency selective channel without considering edge subcarriers in figure 3 . For a sliding window size of 11, the MSE in UFMC systems are $0.7 \mathrm{~dB}$ larger than that in OFDM systems.

\section{RESULTS}

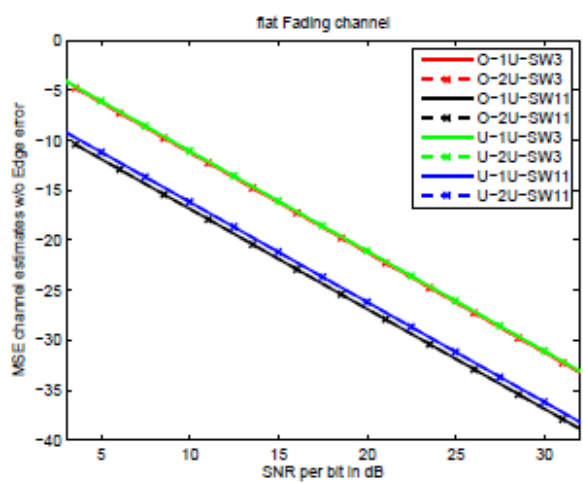

Fig. 3: Performance of Multiuser channel estimator for frequency selective channel without considering edge subcarriers for DFT- sequences.

Next, Simulations are carried out and the results are shown in Fig. 4 for the case of flat-fading channels for OFDM and UFMC systems with different edge subcarrier treatments and sliding window size of 11 .

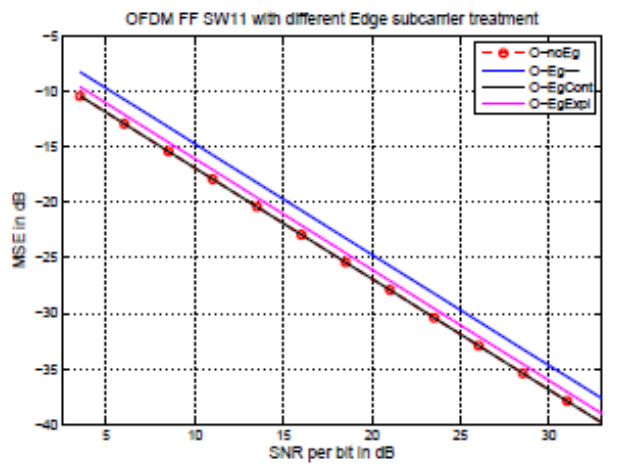

Fig. 4: Comparison of various edge subcarrier techniques in OFDM frequency selective channel model.

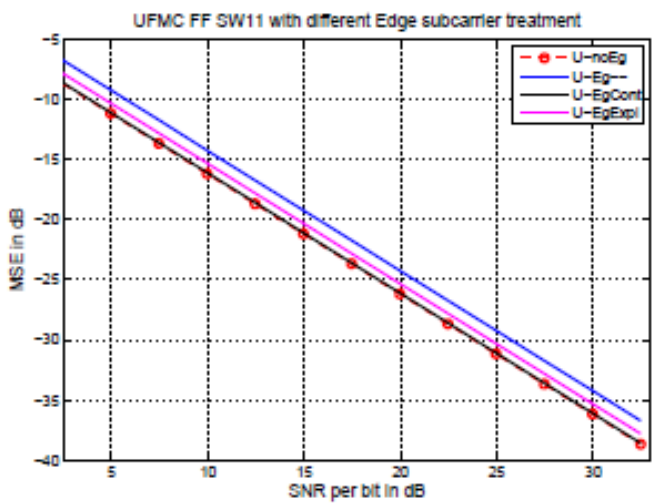

Fig. 5: Comparison of various edge subcarrier techniques in OFDM frequency selective channel model.
Next, the performance among various pilots for flatfading [6] channel model is compared for a window size of 3 in Fig.5. Simulation results show that orthogonal DFTsequence has better performance because of its orthogonality.

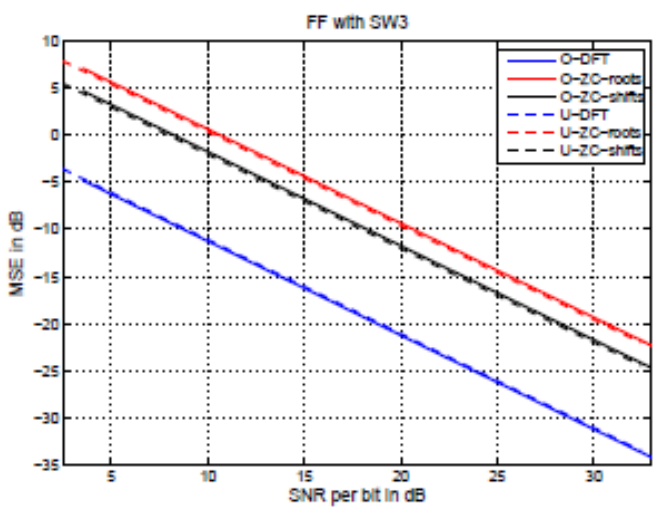

Fig.6: Performance comparison of various pilots in frequency selective channel model.

\section{CONCLUSIONS}

In this paper, synchronization (pilot-based and cyclic prefix- based) is analyzed and investigated. From simulation results, it is observed that pilot-based synchronization method outperforms cyclic prefix- based synchronization with little addend in computational complexity. Further channel estimation along with pilot sequences are studied and investigated for OFDM and UFMC systems. Simulation results are provided for performance comparison of OFDM and UFMC systems in flat fading channel model. These results show that better performance gains were achieved for UFMC systems for the investigated flat-fading channel model.

\section{REFERENCES}

1. M. Elkourdi, B. Peköz, E. Güvenkaya, and H. Arslan, "Waveform design principles for 5G and beyond," in 2016 IEEE 17th Annua Wireless and Microwave Technology Conference (WAMICON), Apr. 2016, pp. 1-6.

2. J. G. Proakis, Digital Communications, 4th ed. McGraw-Hill, 2001

3. T. S. Rappaport, Wireless Communications: Principles and Practice, 2nd ed. Prentice Hall, 2002.

4. B. Farhang-Boroujeny, "OFDM versus Filter Bank Multicarrier," IEEE Signal Processing Magazine, vol. 28, no. 3, pp. 92-112, May 2011.

5. ASahin, I. Guvenc, and H. Arslan, "A survey on multicarrier communications: prototype filters, lattice structures, and implementation aspects," IEEE Communications Surveys Tutorials, vol. 16, no. 3, pp. 1312-1338, 2014.

6. Xiaojie Wang, "Channel Estimation and Equalization for 5G Wireles Communication Systems," PhD thesis, 2014

7. Hidalgo Stitz, T. (2010) Filterbank Techniques for the Physical Layer in Wireless Communications. Ph.D. Theses, Tampere University of Technology Publications, Tampere, 919 p.

8. Patteti Krishna, "Spectral Efficiency Analysis of Multicarrier Scheme for 5G Communications", International Journal of Recent Technology and Engineering (IJRTE), Volume-7, Issue-6, March 2019, pp: 16821686 . 